\title{
Autosomal genetic variation is associated with DNA methylation in regions variably escaping $X$ - chromosome inactivation
}

René Luijk ${ }^{1}$, Haoyu Wu ${ }^{2}$, Cavin K Ward-Caviness ${ }^{3}$, Eilis Hannon ${ }^{4}$, Elena Carnero-Montoro ${ }^{5,22}$, Josine L. Min ${ }^{6,7}$, Pooja Mandaviya ${ }^{8,9}$, Martina Müller-Nurasyid (10) 10,11,12, Hailiang Mei², Silvere M. van der Maarel ${ }^{2}$, BIOS Consortium ${ }^{\#}$, Caroline Relton ${ }^{6}$, Jonathan Mill ${ }^{4}$, Melanie Waldenberger ${ }^{3,13}$, Jordana T. Bell ${ }^{5}$, Rick Jansen (10 ${ }^{14}$, Alexandra Zhernakova ${ }^{15}$, Lude Franke (D) ${ }^{15}$, Peter A.C. 't Hoen (1) ${ }^{2}$, Dorret I. Boomsma ${ }^{16}$, Cornelia M. van Duijn ${ }^{17}$, Marleen M.J. van Greevenbroek ${ }^{18,19}$, Jan H. Veldink ${ }^{20}$, Cisca Wijmenga ${ }^{15}$, Joyce van Meurs ${ }^{8}$, Lucia Daxinger ${ }^{2}$, P. Eline Slagboom', Erik W. van Zwet $^{21} \&$ Bastiaan T. Heijmans (i) $^{1}$

$X$-chromosome inactivation $(\mathrm{XCl})$, i.e., the inactivation of one of the female $\mathrm{X}$ chromosomes, restores equal expression of $\mathrm{X}$-chromosomal genes between females and males. However, $\sim 10 \%$ of genes show variable degrees of escape from $\mathrm{XCl}$ between females, although little is known about the causes of variable XCI. Using a discovery data-set of 1867 females and 1398 males and a replication sample of 3351 females, we show that genetic variation at three autosomal loci is associated with female-specific changes in X-chromosome methylation. Through cis-eQTL expression analysis, we map these loci to the genes SMCHD1/METTL4, TRIM6/HBG2, and ZSCAN9. Low-expression alleles of the loci are predominantly associated with mild hypomethylation of $\mathrm{CpG}$ islands near genes known to variably escape $\mathrm{XCl}$, implicating the autosomal genes in variable $\mathrm{XCl}$. Together, these results suggest a genetic basis for variable escape from $\mathrm{XCl}$ and highlight the potential of a population genomics approach to identify genes involved in $\mathrm{XCl}$.

\footnotetext{
${ }^{1}$ Molecular Epidemiology, Department of Biomedical Data Sciences, Leiden University Medical Center, Leiden 2333 ZC, The Netherlands. ${ }^{2}$ Department of Human Genetics, Leiden University Medical Center, Leiden 2333 ZC, The Netherlands. ${ }^{3}$ Institute of Epidemiology II, Helmholtz Zentrum München, Neuherberg 85764 Oberschleißheim, Germany. ${ }^{4}$ University of Exeter Medical School, Exeter EX4 4QD, UK. ${ }^{5}$ Department of Twin Research \& Genetic Epidemiology, King's College London, London SE1 7EH, UK. ${ }^{6}$ MRC Integrative Epidemiology Unit, University of Bristol, Bristol BS8 1TH, UK. ${ }^{7}$ Bristol Medical School, University of Bristol, Bristol BS8 1UD, UK. ${ }^{8}$ Department of Internal Medicine, Erasmus University Medical Center, Rotterdam 3015 CE, The Netherlands. ${ }^{9}$ Department of Clinical Chemistry, Erasmus University Medical Center, Rotterdam 3015 CE, The Netherlands. ${ }^{10}$ DZHK (German Centre for Cardiovascular Research), partner site: Munich Heart Alliance, Munich 80802, Germany. ${ }^{11}$ Institute of Genetic Epidemiology, Helmholtz Zentrum München German Research Center for Environmental Health, Neuherberg D-85764, Germany. ${ }^{12}$ Department of Medicine I, University Hospital Munich, LudwigMaximilians-University, Munich 80336, Germany. ${ }^{13}$ Research Unit of Molecular Epidemiology, Helmholtz Zentrum München, Neuherberg D-85764, Germany. ${ }^{14}$ Department of Psychiatry, VU University Medical Center, Neuroscience Campus Amsterdam, Amsterdam 1081 HV, The Netherlands. ${ }^{15}$ Department of Genetics, University of Groningen, University Medical Centre Groningen, Groningen 9713 AV, The Netherlands. ${ }^{16}$ Department of Biological Psychology, Vrije Universiteit Amsterdam, Neuroscience Campus Amsterdam, Amsterdam 1081 TB, The Netherlands. ${ }^{17}$ Department of Epidemiology, Genetic Epidemiology Unit, ErasmusMC, Rotterdam 3015 GE, The Netherlands. ${ }^{18}$ Department of Internal Medicine, Maastricht University Medical Center, Maastricht 6211 LK, The Netherlands. ${ }^{19}$ School for Cardiovascular Diseases (CARIM), Maastricht University Medical Center, Maastricht 6229 ER, The Netherlands. ${ }^{20}$ Department of Neurology, Brain Center Rudolf Magnus, University Medical Center Utrecht, Utrecht 3584 CG, The Netherlands. ${ }^{21}$ Medical Statistics, Department of Biomedical Data Sciences, Leiden University Medical Center, Leiden 2333 ZC, The Netherlands. ${ }^{22}$ Present address: Pfizer University of Granada - Andalusian Government Center for Genomics and Oncological Research (GENYO), Granada 18016, Spain. Correspondence and requests for materials should be addressed to.B.T.H. (email: b.t.heijmans@lumc.nl). A full list of consortium members appears at the end of the paper.
} 
T $\mathrm{o}$ achieve dosage equivalency between male and female mammals, one of two X-chromosomes is silenced early in female embryonic development resulting in one inactive (Xi) and one active (Xa) copy of the $\mathrm{X}$-chromosome ${ }^{1}$. While the $\mathrm{Xi}$-linked gene XIST is crucial for the initiation of X-chromosome inactivation (XCI), autosomal genes appear to be critically involved in XCI establishment and maintenance ${ }^{2}$. An abundance of repressive histone marks ${ }^{3-5}$ and DNA methylation ${ }^{6,7}$ throughout $\mathrm{XCI}$ on $\mathrm{Xi}$ is in line with a prominent role of epigenetic regulation in both phases. However, the $\mathrm{Xi}$ is not completely inactivated. With an estimated $15 \%$ of X-chromosomal genes consistently escaping XCI, and an additional $10 \%$ escaping $\mathrm{XCI}$ to varying degrees ${ }^{8,9}$, escape from XCI is fairly common in humans ${ }^{10-12}$, much more so than in mice ${ }^{13}$. Genes escaping XCI are characterized by distinct epigenetic states ${ }^{14}$ and are thought to be associated with adverse outcomes, including mental impairment ${ }^{12-14}$.

In the mouse, an example of an autosomal gene involved in $\mathrm{XCI}$ is Smchd1. Smchd1 is an epigenetic repressor and plays a critical role in the DNA methylation maintenance of XCI in mice ${ }^{15,16}$. However, in humans, in-depth knowledge on the role of autosomal genes in XCI maintenance is lacking, despite earlier in vitro ${ }^{17}$ efforts. Furthermore, the mechanisms underlying variable $\mathrm{XCI}$, a common feature of human $\mathrm{XCI}^{8,9}$, are unknown.

Here, we report on the identification of four autosomal loci associated with female-specific changes in X-chromosome DNA methylation using a discovery set of 1867 females and 1398 males, and replication of three of these loci in an independent replication set consisting of 3351 female samples. The replicated loci map to the genes SMCHD1/METTL4, TRIM6/HBG2, and ZSCAN9 through eQTL analysis. All three preferentially influenced the methylation of $\mathrm{CpGs}$ located in $\mathrm{CpG}$ islands (CGI) near genes known to variably escape XCI between individuals, providing evidence for a genetic basis of this phenomenon.

\section{Results}

Identifying female-specific genetic effects on $\mathrm{X}$-methylation. To identify genetic variants involved in XCI, we employed a global test approach ${ }^{18}$ to evaluate the association of 7,545,443 autosomal genetic variants with DNA methylation at any of $10,286 \mathrm{X}$-chromosomal CpGs measured in whole blood of 1867 females (Supplementary Data 1) using the Illumina 450k array (see 'Methods'). The analysis was corrected for covariates, including cell counts, age, and batch effects. We identified 4504 individual variants representing 48 independent loci associated with X-chromosomal methylation in females (Wald $P<5 \times 10^{-8}$, Fig. 1 and Supplementary Fig. 1), each defined by the most strongly associated variant (as reflected by the lowest $P$-value), termed the sentinel variant. Of the 48 sentinel variants corresponding to these 48 loci, 44 were also associated with $\mathrm{X}$-chromosomal methylation in males $(N=1398$, Supplementary Data 1 , Supplementary Data 2; Wald $P<1.1 \times 10^{-6}$ ) indicating that the associations were unrelated to XCI. The four remaining variants did not show any indication for an effect in males (Wald $P>0.19$ ) while they did show strong, widespread, and consistent same-direction effects across the X-chromosome in females (Supplementary Data 2, Supplementary Data 3, Supplementary Fig. 2). Formally testing for a genotype by sex interaction revealed significant interaction effects for three of the four variants. The rs140837774, rs139916287, and rs1736891 variants with evidence for an interaction $\left(P_{\text {interaction }}<5.9 \times 10^{-4}\right)$ mapped to the SMCHD1/ METTL4, TRIM6/HBG2, and ZSCAN9 loci, respectively, (see 'Methods'). The remaining variant $\operatorname{rs} 73937272\left(P_{\text {interaction }}=0.88\right)$ mapped near the ZNF616 gene. Finally, we evaluated whether the effect of the autosomal loci was influenced by genetic variation on $\mathrm{X}$, but this did not change the results (Supplementary Data 4, see 'Methods').

To establish the validity and stability of the analyses, we first investigated whether any of the associations were due to confounding by cellular heterogeneity. Therefore, we directly tested for an association between the four identified sentinel variants and the observed red and white blood cell counts. This did not result in any significant association (Supplementary Fig. 3). Furthermore, we determined that none of the four identified variants are among the variants known to affect blood composition $^{19,20}$. Vice versa, genetic variants known to affect blood cell counts also did not show an association with Xchromosomal methylation in our data (Supplementary Fig. 4, Supplementary Data 5). Re-testing the effects of the four sentinel variants while adjusting for nearby blood composition-associated SNPs $(<1 \mathrm{Mb})$ did not influence the results (Supplementary Data 6, see 'Methods'). Second, we addressed unknown confounding by including latent factors as covariates in our models, estimated in the methylation data using software for estimation and adjustment of unknown confounders in highdimensional data ${ }^{21}$ (see 'Methods'). Re-testing the four sentinel variants without these latent factors did not change the results (Supplementary Data 6). We conclude that the effects of the four variants identified in the discovery data are stable and not confounded by cellular heterogeneity or other, unknown, factors.

Finally, we tested the four sentinel variants in an additional 3351 unrelated female samples (see 'Methods' and Supplementary Data 7), and successfully replicated the rs140837774, rs139916287, and rs1736891 variants (Bonferroni corrected, $P_{\text {adj }}=0.0096, P_{\text {adj }}=2.4 \times 10^{-4}$, and $P_{\text {adj }}=2.2 \times 10^{-3}$, respectively). The rs73937272 variant $\left(P_{\mathrm{adj}}=1\right)$, which also lacked a sex-genotype interaction effect in the discovery set, was not replicated. In further analyses, we focussed on the three replicated loci.

Exploration of genetic loci affecting $\mathrm{X}$-methylation. The sentinel variant $\mathrm{rs} 140837774$ is an AATTG insertion/deletion variant $(\mathrm{MAF}=0.49)$ on chromosome 18 , located in intron 26 of SMCHD1 (Supplementary Fig. 2), a gene known to be critically involved in XCI in mice ${ }^{15,22-25}$. In addition, SMCHD1 mutations affect the methylation levels of the D4Z4 repeat in humans, playing an important role in facioscapulohumeral dystrophy 2 (FSHD2) $^{26}$. To link rs140837774 to a nearby gene we performed a cis-eQTL analysis using RNA-seq data from the 1867 females in the discovery set of our study $(250 \mathrm{~Kb}$ upstream and downstream of the sentinel variant, Supplementary Data 8). We found that the deletion was strongly associated with decreased SMCHD1 expression (Fisher's $P=1.8 \times 10^{-10}$, regression coefficient $=$ $-0.13)$ and increased expression of the methyltransferase METTL4, albeit weaker (Wald $P=4.9 \times 10^{-4}$, regression coefficient $=0.04)$. METTL4 is a highly conserved gene ${ }^{27,28}$, involved in the mRNA modification $\mathrm{N}^{6}$-methyladenosine (reviewed in ref. ${ }^{29}$ ), which plays an important role in epigenetic regulation in mammals ${ }^{30}$

The SMCHD1/METTL4 variant was associated with altered methylation levels of 57X-chromosomal CpGs in females (FDR < 0.05 , Fig. 2a, Supplementary Data 9). The deletion (the low SMCHD1 expression allele) was associated with hypomethylation at 56 of those X-chromosomal CpGs $\left(98.2 \%\right.$, binomial $P=8.5 \times 10^{-13}$ Fig. 2a), consistent with $X$-hypomethylation in female mice deficient for $S M C H D 1^{25}$. The mean effect size was $1 \%$ per rare allele (ranging from 0.27 to $2.34 \%$, Supplementary Fig. 5), with the mean methylation values per $\mathrm{CpG}$ ranging from 2.6 to $55 \%$ (average methylation at these $56 \mathrm{CpGs}$ is $23.6 \%$ ). 


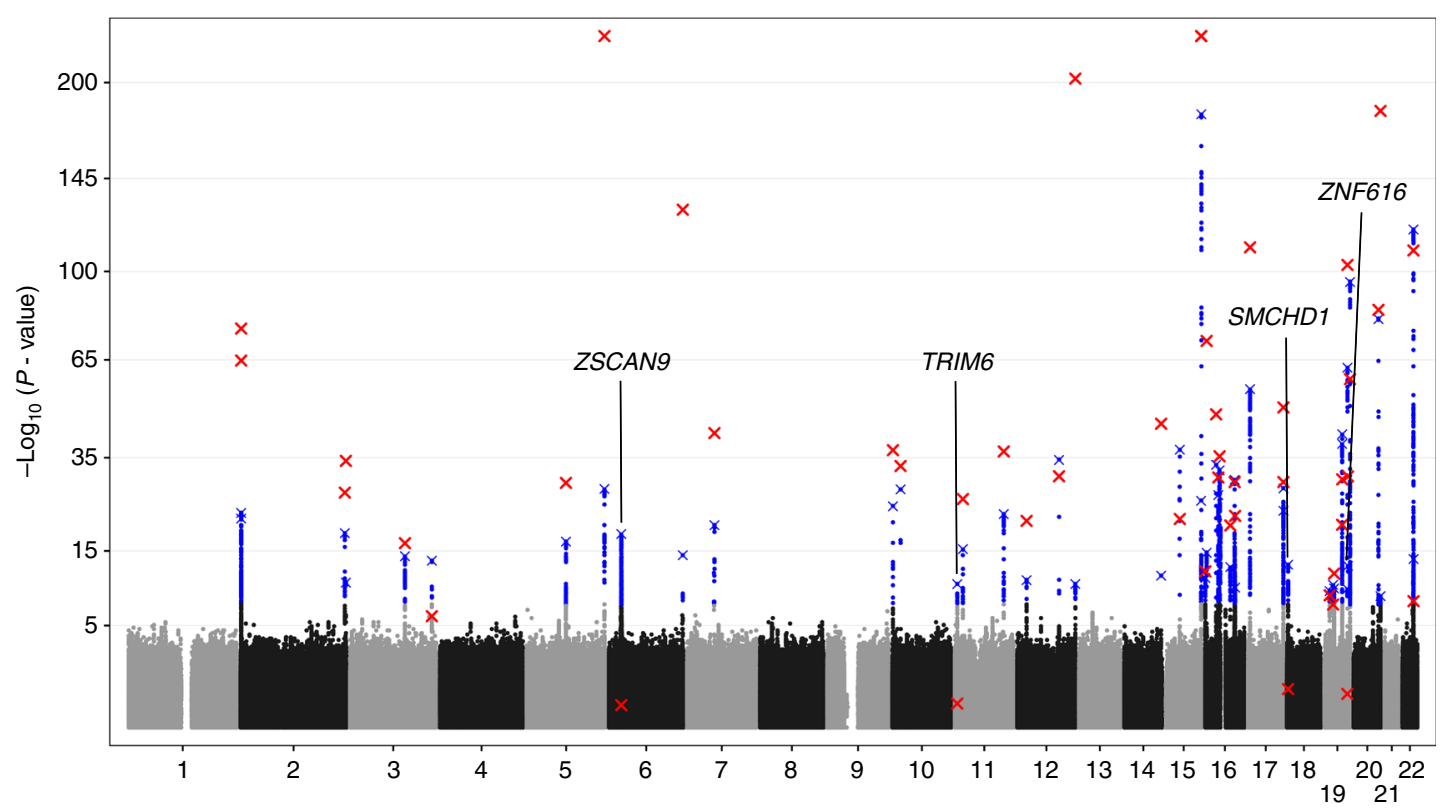

Fig. 1 Manhattan plot showing all tested autosomal SNPs for an overall effect on X-chromosomal methylation in females. Significant associations are depicted in blue (Wald $P<5 \times 10^{-8}$ ). The sentinel variant per independent locus is indicated with a blue cross. Testing the effects of these 48 sentinel variants in males, we found 44 replicated in males (Wald $P<1.1 \times 10^{-6}$, red cross), whereas the other 4 loci were female-specific, as they clearly lacked an effect in males (Wald $P>0.19$ )

a

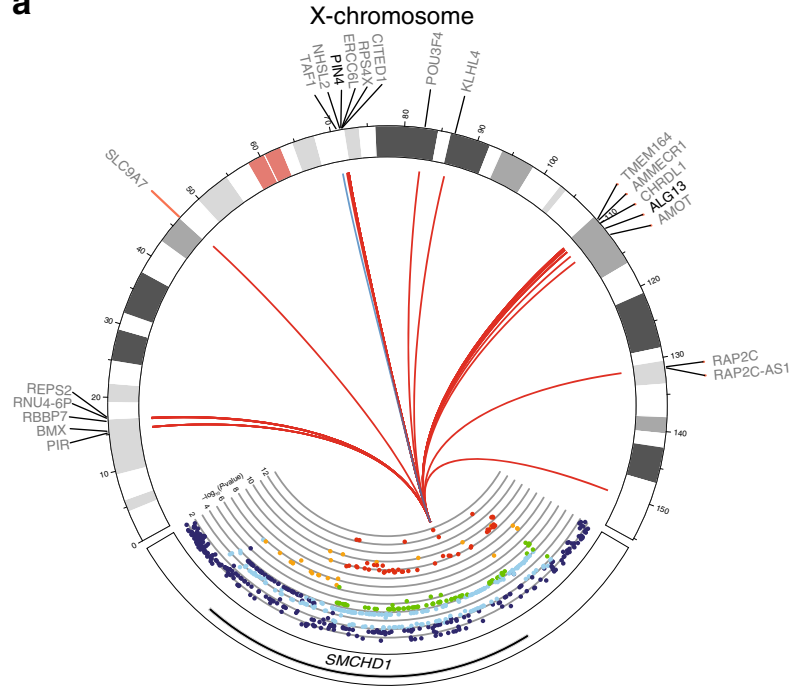

b

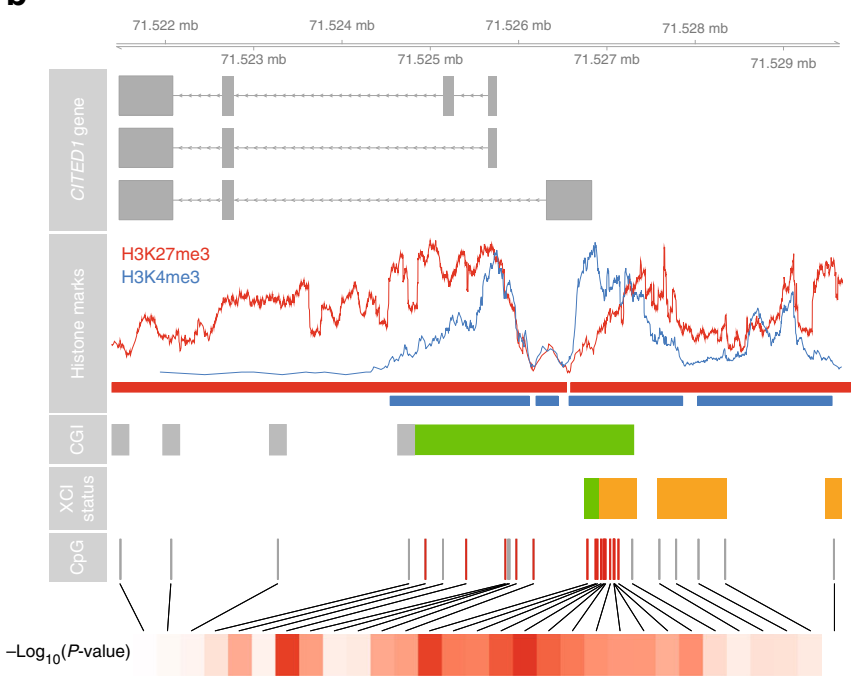

Fig. 2 The SMCHD1/METTL4 locus associates with DNA methylation at X-chromosomal and autosomal regions. a Plot showing the SMCHD1/METTL4 locus and the effects it has on the X-chromosome. The colors in the SMCHD1/METTL4 locus indicate LD (red: $R^{2} \geq 0.8$; orange: $0.6 \leq R^{2}<0.8 ;$ green: $0.4 \leq R^{2}<$ 0.6 ; light blue: $0.2 \leq R^{2}<0.4$; dark blue: $\left.R^{2} 0.6 \leq 0.2\right)$. The $y$-axis shows the - $\log _{10}(P$-value) of the association with overall $\mathrm{X}$-chromosomal methylation. The line colors in the Circos plot indicate the direction of the effect (red: hypomethylation, blue: hypermethylation). The plot of the SMCHD1/METTL4 locus mostly shows moderate to high LD and covers most of the SMCHD1 gene. The deletion of rs140837774 is associated with both downregulation of SMCHD1 and upregulation of METTL4 (see main text). Its effects on X-chromosomal methylation are abundant and consistent: the deletion of rs 140837774 is associated with hypomethylation at $98.2 \%$ of all associated $\mathrm{CpGs}$ ( $56 \mathrm{CpGs}$, red lines, mean effect size $1 \%$ per allele). Hypomethylation of $\mathrm{CpGs}$ near two genes known to escape $X C I$ to varying degrees ${ }^{12}$ (PIN4 and $A L G 13$, shown in bold) is associated with increased expression of these two genes. $\mathbf{b}$ Example of CpG island (CGI) in the CITED1 gene (first row) associated with the SMCHD1/METTL4 locus. The CpGs associated with the SMCHD1/METTL4 locus (fifth row, indicated by red lines) are overrepresented in regions characterized by both active (blue) and repressive (red) histone marks (second row, red and blue bars, two-fold enrichment, Fisher's $P=1.5 \times 10^{-12} ; 6.9$-fold enrichment, Fisher's $P=1.5 \times 10^{-12}$ ), are often located in $C p G$ islands (third row, green bar, 11.3-fold enrichment, Fisher's $P=2.5 \times 10^{-14}$ ), and regions known to variably escape X-chromosome inactivation ${ }^{10}$ (fourth row, orange bars, 21.4 -fold enrichment, Fisher's $P=3.7 \times 10^{-18}$ ). The bottom row indicates the strength of the associations in terms of $-\log _{10}(P$-value) (dark red indicates strong associations) 
Compared to all X-chromosomal CpGs in our data, the associated $\mathrm{X}$-chromosomal $\mathrm{CpGs}$ were strongly overrepresented in CGI (50 out of 57 CpGs, 11.3-fold enrichment, binomial $P=$ $2.5 \times 10^{-14}$, Fig. 2b), in line with SMCHD1's role in $\mathrm{X}$ chromosomal CGI methylation ${ }^{24}$. Data on chromatin marks in blood $^{31}$ (see 'Methods') revealed a strong overrepresentation of the associated X-chromosomal CpGs in regions bivalently marked by the active histone mark H3K4me3 (47 CpGs, 8.2-fold enrichment, Fisher's $\left.P=1.5 \times 10^{-12}\right)$, and the repressive mark H3K27me3 (38 CpGs, 6.9-fold enrichment, Fisher's $P=1.5 \times 10^{-12}$ ), as compared to all $\mathrm{X}$-chromosomal $\mathrm{CpGs}$ in our data. In agreement with this, we observed a 16.9-fold enrichment for CpGs overlapping bivalent/poised transcription start sites (TSSs) (35/57 CpGs, Fisher's $P=4.3 \times 10^{-23}$ ) using predicted chromatin segmentations $^{31}$, possibly reflecting the mixed signals from both the active and inactive $\mathrm{X}$ chromosomes underlying these chromatin segmentations. Strikingly, annotation by the degree of escape for 489 TSSs in 27 different tissues, and specifically whole blood ${ }^{10}$ (see 'Methods'), revealed a strong overrepresentation of CpGs located near TSSs variably escaping XCI (22 CpGs, 21.4-fold enrichment, Fisher's $P=3.7 \times 10^{-18}$, Fig. $2 \mathrm{~b}$ ). Only a modest enrichment for associated CpGs in fully escaping XCI regions (15 CpGs, 4.2-fold enrichment, Fisher's $P=4.5 \times 10^{-5}$ ) and an underrepresentation of associated CpGs in inactivated regions (7 CpGs, 28.6-fold depletion, Fisher's $P=2.2 \times 10^{-23}$ ) was observed.

Further supporting a link with variable XCI, we observed that $\mathrm{X}$-chromosomal CpGs were associated with differential expression of the nearby genes ( $<250 \mathrm{~Kb}$, see 'Methods') $A L G 13$ and PIN4 (see 'Methods', Supplementary Data 10) both known to variably escape $\mathrm{XCI}^{12}$. While a strong eQTL effect and a clear biologically relevant link with XCI mainly implies SMCHD1 in Xchromosomal hypomethylation (insertion of rs140837774), an eQTL effect for METTL4, although slightly weaker, leaves open a possible role for METTL4 in XCI, given its role in the mRNA modification $\mathrm{N}^{6}$-methyladenosine.

Using both female and male samples $(N=3265$, Supplementary Fig. 6) to investigate associations of genetic variation at the SMCHD1/METTL4 locus with autosomal methylation in trans $(>5 \mathrm{Mb})$, we found that the SMCHD1/METTL4 variant was associated with $20 \mathrm{CpGs}$ mapping to the HOXD10, HOXC10, and HOXC11 genes of the HOXD and HOXC clusters located on chromosomes 2 and 12, as well as to the large protocadherin beta $(P C D H \beta)$ and gamma $(P C D H \gamma)$ clusters on chromosome 5 (FDR $<0.05$, Supplementary Fig. 7, Supplementary Data 11), all known SMCHD1 targets ${ }^{25,32}$

The second of the three sentinel variants, sentinel SNP rs139916287 (MAF $=0.07)$, is located in intron 4 of the HBG2 gene on chromosome 11, in the $\beta$-globin locus (rs139916287, chromosome 11, Supplementary Fig. 2B). The rare allele of the sentinel variant was associated with decreased expression of both the HBG2 and TRIM6 genes ( $\mathrm{T}$ allele; Wald $P=5.3 \times 10^{-7}$, regression coefficient $=-130.55$; Wald $P=9.8 \times 10^{-5}$, regression coefficient $=-0.05$; Supplementary Data 8 ), based on cis-eQTL mapping testing genes up to $250 \mathrm{~kb}$ upstream, and downstream of the sentinel variant (Supplementary Data 8). While HBG2 showed higher expression levels and a stronger eQTL effect in our data, TRIM6 has been shown to bind XIST ${ }^{33}$, and contributes to the maintenance of pluripotency in mouse embryonic stem cells ${ }^{34}$, making TRIM6 a strong candidate for explaining our observations. Associating the TRIM6/HBG2 variant with Xchromosomal methylation, we found 276 associated $\mathrm{X}$ chromosomal $\mathrm{CpG}$ sites $(\mathrm{FDR}<0.05$, Fig. 3a, Supplementary Data 9). The rare allele ( $\mathrm{T}$ allele) was associated with hypomethylation at 258 of those CpGs $(93.5 \%$, binomial $P=$ $6.3 \times 10^{-47}$ ), where mean effect size at these $258 \mathrm{CpGs}$ is $1.6 \%$ per $\mathrm{T}$ allele, ranging from $0.15 \%$ to $4.25 \%$ (Supplementary Fig. 5). a

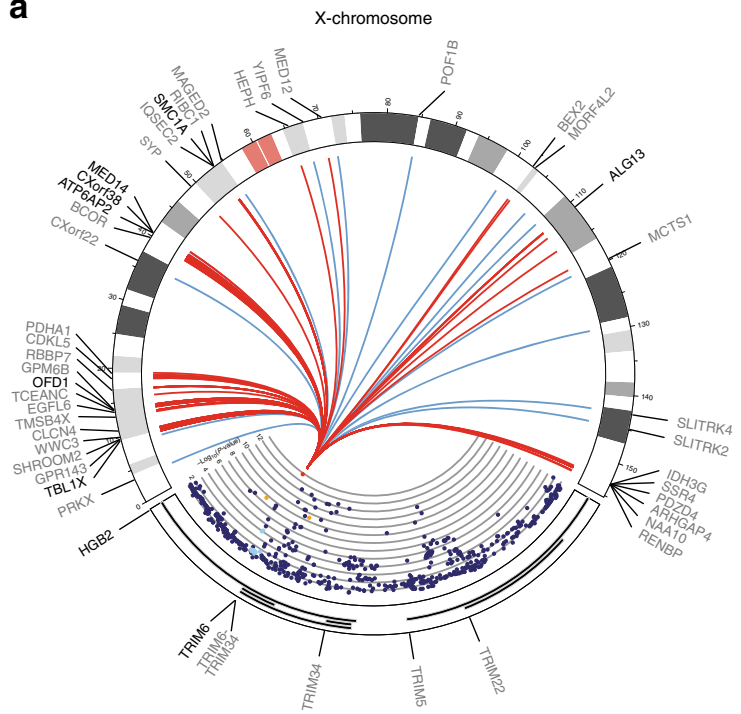

b

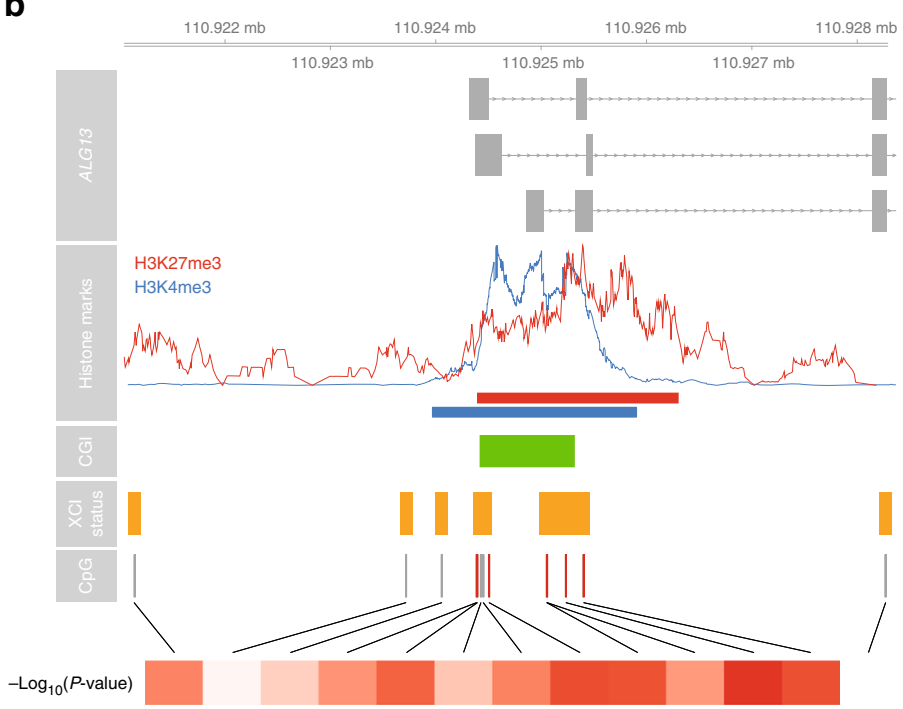

Fig. 3 The TRIM6/HBG2 locus is associated with DNA methylation at X-chromosomal regions. a Plot showing the TRIM6/HBG2 locus and the widespread effects it has on the X-chromosome. The colors in the TRIM6/HBG2 locus indicate LD (red: $R^{2} \geq 0.8$; orange: $0.6 \leq R^{2}<0.8$; green: $0.4 \leq R^{2}<0.6$; light blue: $0.2 \leq R^{2}<0.4$; dark blue: $\left.R^{2} 0.6 \leq 0.2\right)$. The $y$-axis shows the $-\log _{10}(P$-value) of the association with overall $\mathrm{X}$-chromosomal methylation. The line colors in the Circos plot indicate the direction of the effect (red: hypomethylation, blue: hypermethylation). The T allele of its sentinel variant rs 139916287 is associated with upregulation of HBG2, downregulation of TRIM6 (both shown in bold), and hypomethylation at 258 of the 276 associated CpGs ( $93.5 \%$, red lines, mean effect size $1.6 \%$ per allele). X-chromosomal genes whose expression levels were associated with methylation levels of nearby $\mathrm{CpGs}$ are shown in bold. $\mathbf{b}$ Example of $\mathrm{CpG}$ island (CGI) in the ALG13 gene associated with the TRIM6/HBG2 locus. The enrichments of CpGs in certain genomic regions are similar to those found for the SMCHD1/METTL4 locus. Most notably, the associated CpGs are also overrepresented in regions known to variably escape $\mathrm{X}$-chromosome inactivation ${ }^{10}$ (fourth row, orange bars, 8.8-fold enrichment, Fisher's $P=2.1 \times 10^{-20}$ ) 
Similar to the SMCHD1/METTL4 variant, associated CpGs were overrepresented in CGIs (199 CpGs, 4.2-fold enrichment, Fisher's $\left.P=1.6 \times 10^{-29}\right)$, and enriched in regions characterized by H3K27me3 (208 CpGs, 10.5-fold enrichment, Fisher's $P=$ $3.5 \times 10^{-77}$ ) and $\mathrm{H} 3 \mathrm{~K} 4 \mathrm{me} 3^{31}$ (217 CpGs, 6.4-fold enrichment, Fisher's $P=5.5 \times 10^{-46}$, Fig. $3 \mathrm{~b}$ ). The associated CpGs were again strongly overrepresented in genomic regions variably escaping $\mathrm{XCI}$ in an external set of whole blood samples ${ }^{10}$ (see 'Methods', 39 CpGs, 8.8-fold enrichment, Fisher's $\left.P=2.1 \times 10^{-20}\right)$, to a lesser extent present in regions consistently escaping XCI (61 CpGs, 6.8-fold enrichment, Fisher's $P=2.2 \times 10^{-23}$ ), and underrepresented in repressed regions (39 CpGs, 15.2-fold depletion, Fisher's $\left.P=1.9 \times 10^{-50}\right)$.

In addition, many genes known to variably escape $\mathrm{XCI}^{12}$, annotated to CpGs associated with TRIM6/HBG2 genetic variation (ALG13, ATP6AP2, CXorf38, MED14, SMC1A, TBL1X, Supplementary Data 10$)$. Similar to $S M C H D 1 / M E T T L 4$, these results suggest a role for the TRIM6/HBG2 locus in variable escape from XCI.

The sentinel SNP of the third identified locus (rs1736891, MAF $=0.38$ ) was associated with the expression of several nearby genes annotated as zinc fingers (Supplementary Data 8), but most strongly with downregulation of the expression of the nearby transcription factor $^{35}$ ZSCAN9 gene located on chromosome 6, based on ciseQTL mapping in our own data (Wald $P=2.5 \times 10^{-49}$, Supplementary Data 8 ). The sentinel SNP was significantly associated with $19 \mathrm{X}$-chromosomal CpGs in females (FDR $<0.05$, Supplementary Fig. 8, Supplementary Data 9), all located in the same CGI: the highexpression A allele of rs1736891 was associated with mild hypomethylation of all 19 CpGs (Fisher's $P=3.6 \times 10^{-5}$, mean effect size $1.3 \%$ per allele, Supplementary Fig. 5). There was an overlap of in the CpGs associated with the sentinel variants of the ZSCAN9 and the SMCHD1/METTL4 locus, although the two loci are located on different chromosomes (chromosomes 6 and 18, respectively, Supplementary Fig. 2). These associations were statistically independent from each other (i.e., additive), as all identified loci were identified using conditional analyses (see 'Methods'). Specifically, 17 out of 19 CpGs (89.5\%) were also targeted by the SMCHD1/METTL4 locus, and all 19 CpGs show consistent opposite effects for both loci (Supplementary Fig. 9). Similar to the SMCHD1/METTL4 locus, the ZSCAN9 locus also associated with autosomal DNA methylation in trans $(>5 \mathrm{Mb})$. However, none of the autosomal CpGs overlapped between the two loci (Supplementary Data 11).

\section{Discussion}

Here, we identify three autosomal genetic loci with femalespecific effects on X-chromosomal methylation in humans (SMCHD1/METTL4, TRIM6/HBG2, and ZSCAN9), all of which were associated with altered expression of autosomal genes in cis. Furthermore, all three loci were consistently associated with mild hypomethylation of CpGs overrepresented in CGI of Xchromosomal regions known to variably escape XCI in whole blood $^{10,12}$. The former finding extended to 26 other tissues ${ }^{10}$, suggesting a cross-tissue genetic basis for variable escape from XCI. We observed a striking underrepresentation of affected CpGs in fully inactivated CGIs. This may be due to the tightly regulated nature of these regions. Methylation of these CpGs may be impervious to the impact of autosomal genetic variation or effects may be substantially weaker requiring much larger data sets to detect them.

While most of the previous work on XCI was done using mouse models and established a critical role for SMCHD1 in $\mathrm{XCI}^{15,23,25}$, we here confirm the role of the SMCHD1/METTL4 locus in XCI in humans and highlight its impact on variable escape from XCI. This phenomenon has not been previously described in mice, perhaps due to the lack of genetic variability in the often inbred mice, leading to less (variable) escape from XCI than occurs in humans 8,9 . We also observed associations of the SMCHD1/METTL4 locus with known autosomal SMCHD1 targets $^{25,32}$, most notably the protocadherin clusters ${ }^{36}$. Interestingly, similar to the X-chromosome, the expression of the clustered protocadherin genes is stochastic and mono-allelic ${ }^{37}$, suggesting a common mechanism.

In addition to the SMCHD1/METTL4 locus, our results indicated a role for the TRIM6/HBG2 locus in XCI. TRIM6 is a strong candidate to influence female $\mathrm{X}$-chromosome methylation because it was reported to bind XIST ${ }^{33}$ and is involved in MYC and NANOG regulation ${ }^{34}$. Similarly, our data suggest a role for the ZSCAN9 locus in variable escape from XCI, as it affects a single CGI that is also targeted by the SMCHD1/METTL4 locus. While this does suggest a role for the two loci in the same pathway, the effects on the X-chromosome were statistically independent.

Given the biological consistency of the findings presented here, and the replication thereof in an independent set of samples, our data support a role of autosomal genetic variants in regulating $\mathrm{Xi}$ methylation in particular at variably escaping regions. However, to definitely demonstrate causality, unequivocally identify the responsible genes, and provide precise insight into the exact underlying mechanisms, in vitro experiments are needed. Importantly, a population genomics approach, like ours, will reveal effects on both XCI establishment and maintenance, which occur during different developmental stages and may involve different molecular pathways. At this point, the exact role of the SMCHD1/METTL4, TRIM6/HBG2, and ZSCAN9 loci during these processes remain to be determined. Therefore, it will be crucial to design experiments that can discriminate between an effect during the establishment and maintenance phases.

In conclusion, variable escape from XCI in humans has a genetic basis and we identified three autosomal loci, one previous implicated in XCI in mice and two new loci, that influence regions that are susceptible to variable escape from XCI by controlling X-chromosomal DNA methylation or correlated epigenetic marks.

\section{Methods}

Discovery cohorts. The Biobank-based Integrative Omics Study (BIOS) Consortium comprises six Dutch biobanks: Cohort on Diabetes and Atherosclerosis Maastricht (CODAM) ${ }^{38}$, LifeLines-DEEP (LLD) ${ }^{39}$, Leiden Longevity Study (LLS) ${ }^{40}$, Netherlands Twin Registry (NTR) ${ }^{41}$, Rotterdam Study (RS $)^{42}$, Prospective ALS Study Netherlands $(\mathrm{PAN})^{43}$. Institutional review boards of all cohorts approved this study (CODAM, Medical Ethical Committee of the Maastricht University; LL, Ethics committee of the University Medical Centre Groningen; LLS, Ethical committee of the Leiden University Medical Center; PAN, Institutional review board of the University Medical Centre Utrecht; NTR, Central Ethics Committee on Research Involving Human Subjects of the VU University Medical Centre; RS Institutional review board (Medical Ethics Committee) of the Erasmus Medical Center). In addition, informed consent was provided by all participants. The data that were analyzed in this study came from 3265 unrelated individuals (Supplementary Data 1). Genotype data, DNA methylation data, and gene expression data were measured in whole blood for all samples. In addition, sex, age, measured cell counts (lymphocytes, neutrophils, monocytes, eosinophils, basophils, and red blood cell counts), and information on technical batches were obtained from the contributing cohorts. The Human Genotyping facility (HugeF, Erasmus MC, Rotterdam, The Netherlands, http://www.glimdna.org) generated the methylation and RNA-sequencing data and supplied information on technical batches.

Genotype data were generated within each cohort. Details on the genotyping and quality control methods have previously been detailed elsewhere (LLD: Tigchelaar et al.; ${ }^{39}$ LLS: Deelen et al. ${ }^{44}$, 2014; NTR: Lin et al.; ${ }^{45}$ RS: Hofman et al.; ${ }^{42}$ PAN: Huisman et al. ${ }^{43}$ ).

For each cohort, the genotype data were harmonized towards the Genome of the Netherlands ${ }^{46}$ (GoNL) using Genotype Harmonizer ${ }^{47}$ and subsequently imputed per cohort using Impute $2^{48}$ and the GoNL reference panel (v5) ${ }^{46}$. We removed SNPs with an imputation info-score below 0.5 , a HWE $P$-value $<10^{-4}$, a call rate below $95 \%$ or a minor allele frequency smaller than 0.01 . These imputation 
and filtering steps resulted in 7,545,443 SNPs that passed quality control in each of the datasets.

A detailed description regarding generation and processing of the gene expression data can be found elsewhere ${ }^{49}$. Briefly, total whole blood was processed using Illumina's Truseq version 2 library preparation kit. Illumina's Hiseq2000 was used for paired-end sequencing. Lastly, CASAVA was used to create read sets per sample, applying Illumina's Chastity Filter. Data generation was done by the Human Genotyping facility (HugeF, ErasmusMC, The Netherlands, see URLs). QC was performed using FastQC (v0.10.1), cutadapt (v1.1 $)^{50}$, and Sickle (v1.2) ${ }^{51}$, after which the sequencing reads were mapped to human genome (HG19) using STAR $(\mathrm{v} 2.3 .0 \mathrm{e})^{52}$.

All common GoNL SNPs (MAF >0.01, http://www.nlgenome.nl/?page_id=9) were masked with $\mathrm{N}$ to avoid reference mapping bias. Read pairs used were those with fewer than nine mismatches, and mapping to fewer than six positions.

Gene expression quantification was determined using base counts (for a detailed description, see ref. ${ }^{49}$ ). The gene definitions used for quantification were based on Ensembl version 71. For data analysis, we used reads per kilobase per million mapped reads (RPKM), and only used protein coding genes with sufficient expression levels (median RPKM $>1$ ), resulting in a set of 10,781 genes. To limit the influence of any outliers still present in the data, the data were transformed using a rank-based inverse normal transformation within each cohort.

The Zymo EZ DNA methylation kit (Zymo Research, Irvine, CA, USA) was used to bisulfite-convert $500 \mathrm{ng}$ of genomic DNA, and $4 \mu \mathrm{l}$ of bisulfite-converted DNA was measured on the Illumina HumanMethylation450 array using the manufacturer's protocol (Illumina, San Diego, CA, USA). Preprocessing and normalization of the data were done as described earlier ${ }^{53}$. Removal of ambiguously mapped probes or probes containing known common genetic variants $^{54}$ were removed, followed by quality control (QC) using MethylAid's ${ }^{55}$ default settings, investigating methylated and unmethylated signal intensities, bisulfite conversion, hybridization, and detection $P$-values. Filtering of individual beta-values was based on detection $P$-value $(P<0.01)$, number of beads available $(\leq 2)$ or zero values for signal intensity. Normalization was done using Functional Normalization ${ }^{56}$ as implemented in the minfi $\mathrm{R}$ package $\mathrm{e}^{57}$, using five principal components extracted using the control probes for normalization. All samples or probes with more than $5 \%$ of their values missing were removed, based on the QC performed using MethylAid. The final dataset consisted of 440,825 probes measured in 3265 samples. Lastly, similar to the RNA-sequencing data, the methylation data were also transformed using a rank-based inverse normal transformation within each cohort, to limit the influence of any remaining outliers while removing any systematic differences in mean methylation between cohorts.

Replication cohorts. Samples were drawn from the Avon Longitudinal Study of Parents and Children (ALSPAC) (Fraser et al. 2013; Boyd et al. 2013). Blood from 1022 mother-child pairs (children at three time points and their mothers at two time points) were selected for analysis as part of Accessible Resource for Integrative Epigenomic Studies (ARIES, http://www.ariesepigenomics.org.uk/) (Relton et al. 2015). Written informed consent has been obtained for all ALSPAC participants. Ethical approval for the study was obtained from the ALSPAC Ethics and Law Committee and the Local Research Ethics Committees. Genotyping and methylation measurements have been previously described ${ }^{58,59}$.

The University College London case-control sample has been described elsewhere ${ }^{60,61}$ but briefly comprises of unrelated ancestrally matched schizophrenia cases recruited from NHS mental health services and controls from the United Kingdom. All controls were screened to exclude the presence of an RDC defined mental disorder, alcohol dependence, or a family history thereof. Informed consent was obtained from all participating subjects, as well as UK National Health Service multicentre and local research ethics approval. Details of DNA methylation and genetic data generation, processing, quality control and normalisation can be found in the original EWAS manuscript ${ }^{61}$.

The Aberdeen case-control sample has been described elsewhere ${ }^{61,62}$ but briefly contains schizophrenia cases and controls who have self-identified as born in the British Isles (95\% in Scotland). Controls were selected based on age, sex, and excluded if they presented with a mental disorder, or one of their first degree relatives, or if they used neuroleptic medication. All subjects gave informed consent. Different ethical committees (both local and multiregional) approved of the study. Details of DNA methylation and genetic data generation, processing, quality control and normalisation can be found in the original EWAS manuscript ${ }^{61}$.

The KORA study (Cooperative health research in the Region of Augsburg) consists of independent population-based samples from the general population living in the region of Augsburg, Southern Germany. Written informed consent has been given by each participant and the study was approved by the local ethical committee. The dataset comprised individuals from the KORA F4 survey (all with genotyping and methylation data available) conducted during 2006-2008.

The TwinsUK cohort recruits monozygotic and dizygotic twins since its establishment in $1992^{63}$, includes over 13,000 twin participants, and is a good representation of the general population in the UK and represents this population with respect to several phenotypes, including disease ${ }^{64}$. As such, it has been used in several epidemiological studies.

The Rotterdam Study (RS) is a prospective, population-based cohort study, investigating chronic diseases in the elderly ${ }^{65}$. The study consists of several cohorts
(RS-I, RS-II, RS-III), totalling 14,926 subjects from the Ommoord district in Rotterdam, The Netherlands. All participants gave informed consent. This study was approved by the Erasmus University Medical Center medical ethics committee.

Discovering female-specific genetic effects on X-methylation. To identify autosomal genetic variants influencing DNA methylation anywhere on the Xchromosome we applied a two-step approach ${ }^{18}$ using 1867 female samples from the replication cohorts for which both genotype data and methylation data were available. We first fitted linear models to test for an association between each autosomal SNP $y_{j}$ and each of 10,286X-chromosomal CpGs $x_{i}$ individually, correcting for known covariates $M$ (cell counts, cohort, age, technical batches-e.g., sample plate and array position) and unknown confounding by including latent factors $U$, estimated using cate ${ }^{21}$, where the eigenvalue difference method implemented in cate suggested an optimal number of three latent factors:

$$
y_{j}=\beta_{i j} x_{i}+\gamma M+\delta U
$$

For each autosomal genetic variant $i$, this approach yields $10,286 P$-values $p_{i j}$. Next, we combined all 10,286 $P$-values corresponding to one genetic variant $i$ into one overall $P$-value ${ }_{P i}$ using the Simes procedure ${ }^{66}$, yielding 7,545,443 $P$-values $P_{i}$, one for each autosomal genetic variant tested. This overall $P$-value per SNP indicates if an autosomal SNP influences DNA methylation anywhere on the Xchromosome, reducing this analysis to a GWAS for X-chromosomal DNA methylation. SNPs with an overall $P$-value $<5 \times 10^{-8}$ were deemed significantly associated with $\mathrm{X}$-chromosomal DNA methylation.

To identify independent effects among the identified variants, we performed iterative conditional analyses. We re-ran the entire above procedure, correcting for the strongest associated sentinel variant, as determined by the lowest overall $P$ value.

$$
y_{j}=\beta_{i j} x_{i}+\gamma M+\delta U+\beta_{\text {top SNP }} x_{\text {sentinel }}
$$

Having identified a new top SNP at the same genome-wide significance level of $P<5 \times 10^{-8}$, we again re-did our analysis, now correcting for two top SNPs. We repeated this process until no new independent effects were identified, which was after 47 such iterations, thus yielding 48 sentinel variants, corresponding to 48 different loci.

Next, to establish the female-specificity of the identified loci on X-chromosomal methylation, we aimed to validate the 48 identified loci in 1398 males from the discovery cohorts for which the same genotype and methylation data were available. Any locus also having an effect in males would then mean that particular locus was not female specific. To do this, we tested the sentinel variant per locus found in females in the exact same way as we did in females, but also testing all SNPs within $1 \mathrm{Mb}$ correlated to the sentinel variant $\left(R^{2} \geq 0.8\right.$ in males). A locus with any SNP having an overall $P$-value $p_{i} \leq 0.05$ in males was not considered to be female-specific, yielding four loci with four corresponding sentinel variants.

Replicating female-specific genetic effects on $\mathbf{X}$-methylation. To replicate the four identified sentinel variants, we used an independent sample of 3351 females from five different replication cohorts (see section Description of replication cohorts), all having genotype and $450 \mathrm{k}$ methylation data available. Similar to the discovery phase, each of four sentinel variants $x_{i}$ was associated with all Xchromosomal CpGs $y_{j}$ in each replication cohort $k$ :

$$
y_{j k}=\beta_{i j k} x_{i k}
$$

each yielding a test-statistic $t_{i j k}$. We then combined the test-statistics corresponding to each genetic variant $i$ and $C p G j$ between each cohort $j$ using Stouffer's weighted $Z$-method (discussed in ref. ${ }^{67}$ ), resulting in one overall $Z$-score $Z_{i j}$ for each variantCpG pair $i, j$ :

$$
Z_{i j}=\frac{\sum_{k} w_{k} t_{j k}}{\sqrt{\sum_{k} w_{k}^{2}}}
$$

where $w_{k}$ indicates the sample size for replication cohort $k$. Converting each overall $Z$-score $Z_{i j}$ to a $P$-value $p_{i j}$, we again used the Simes' procedure ${ }^{66}$ to calculate one overall $P$-value ${ }_{P i}$ per genetic variant $i$, representing the statistical evidence for an association with any $\mathrm{X}$-chromosomal $\mathrm{CpG}$ in the replication cohorts.

Local (cis) expression QTL mapping. In order to map the identified sentinel variants associated with female-specific $\mathrm{X}$-chromosomal methylation to nearby genes, we employed cis-eQTL mapping in the discovery cohorts, where we associated the genotypes of a genetic variant $i$ with the expression levels of genes $j$ in cis $(<250 \mathrm{~Kb})$. Similar to the trans-meQTL mapping for chromosome X, we corrected for known covariates $M$ (i.e., cell counts, cohort, age, technical batches), and unknown confounding $U$ using cate, using an optimal number of latent factors to 
include, as suggested by cate:

$$
y_{j}=\beta_{i j} x_{i}+\gamma M+\delta U
$$

Next, we performed the Bonferroni correction to the corresponding $P$-values $p_{i j}$ to identify genes associated with the genetic variant.

Associating X-methylation with nearby gene expression. To identify genes associated with DNA methylation of nearby CpGs $(<250 \mathrm{~Kb})$, we used a similar model as for trans-meQTL and cis-eQTL mapping. We associated methylation levels of CpGs $x_{i}$ with the observed expression values of a gene $y_{j}$ using a linear model, correcting for covariates $M$ (i.e., cell counts, cohort, age, technical batches):

$$
y_{j}=\beta_{i j} x_{i}+\gamma M
$$

The Bonferroni correction was used to determine significant CpG-gene pairs.

Identifying genetic effects on autosomal DNA methylation. To identify longrange effects $(>5 \mathrm{Mb})$ of a genetic variant on DNA methylation at autosomal CpGs, we performed trans-meQTL mapping using all 3265 samples for which both genotype data and methylation data were available, as we expected these effects to be present in both women and men (Supplementary Fig. 6). For any genetic variant $i$ and CpG $j$, we fitted a linear model correcting for known covariates $M$ (cell counts, cohort, age, technical batches), and unknown confounding $U$ using cate:

$$
y_{j}=\beta_{i j} x_{i}+\gamma M+\delta U
$$

The FDR was controlled within each set of corresponding $P$-values $p_{i j}$, to obtain a list of associated CpGs for a genetic variant $i$.

Testing epistatic effects. To test if the identified autosomal loci have any epistatic effects on X-chromosomal DNA methylation, we corrected the analysis for Xchromosomal cis-meQTLs. We first mapped cis-meQTLs $(<250 \mathrm{~Kb})$ on the Xchromosome by testing all nearby genetic variants for an effect on any of the $\mathrm{X}$ chromosomal CpGs associated with one of the three autosomal loci. For any genetic variant $i$ and $\mathrm{CpG} j$, we fitted a linear model correcting for known covariates $M$ (cell counts, cohort, age, technical batches):

$$
y_{j}=\beta_{i j}^{X} x_{i}+\gamma M
$$

We corrected for multiple testing using the Bonferroni procedure, selecting CpGs harboring cis-meQTLs. Next, we re-tested the effects of the autosomal loci on the X-chromosomal CpGs, but this time correcting for the strongest cis-SNP.

$$
y_{j}=\beta_{i j}^{X}+\beta_{i j}^{\text {auto }} x_{i}+\gamma M
$$

Annotations and enrichment tests. CpGs were annotation using UCSC Genome Browser ${ }^{68}$, histone marks and chromatin states data from the Blueprint Epigenome data $^{69}$, transcription factor binding site (TFBS) data from the Encode Project ${ }^{70}$, and data on regions escaping X-inactivation ${ }^{10}$. All annotations were done based on the location of the CpG site using HG19/GRCh37.

The CGI track from the UCSC Genome Browser was used to map CpGs to CGIs. Shores were defined as the flanking $2 \mathrm{~kb}$ regions. All other regions were defined as non-CGI.

We obtained Epigenomics Roadmap ChIP-seq data on histone marks measured in blood-related cell types (the GM12878 lymphoblastoid cell line, the K562 leukemia cell line, and monocytes). We selected five different histone marks for which data measured in both men and women were available (H3K4me3, H3K4me1, H3K9me3, H3K27me3, H3K27ac). A CpG was said to overlap with any histone mark if it did so in any the of the data sets.

We obtained Epigenomics Roadmap data on the 16 predicted core chromatin states data in blood-related cell types (the GM12878 lymphoblastoid cell line, the K562 leukemia cell line, and monocytes). A CpG was said to overlap with any chromatin state if it did so in any of the available data sets for that histone mark. Likewise, we obtained transcription factor binding data from the Encode Project, using blood-related cell types only (GM08714, GM10847, GM12878, GM12892, GM18505, GM18526, GM18951, GM19099, GM19193).

The degree of escape from X-inactivation for 632 TSSs has previously been established in 27 different tissues ${ }^{10}$. Within each tissue, each TSS was said to fully escape XCI, variably escape XCI, or be subject to XCI. We mapped each Xchromosomal CpG to the nearest such TSS, annotating each CpG with the accompanying scores for each of the 27 tissues. CpGs not in the vicinity of any such TSS (>10 kb, $4698 \mathrm{CpGs)}$ were left unannotated.
In order to determine the enrichment of CpGs for any of the described genomic contexts, we used Fisher's exact test, where the used all X-chromosome CpGs as the background set.

Code availability. $\mathrm{R}$ code is available from https://git.lumc.nl/r.luijk/ ChromosomeX. This repository describes the main analyses done.

\section{Data availability}

Raw data were submitted to the European Genome-phenome Archive (EGA) under accession EGAS00001001077.

Received: 6 February 2018 Accepted: 23 July 2018 Published online: 14 September 2018

\section{References}

1. Lyon, M. F. Gene action in the X-chromosome of the mouse (Mus musculus L.). Nature 190, 372-373 (1961).

2. Galupa, R. \& Heard, E. X-chromosome inactivation: new insights into cis and trans regulation. Curr. Opin. Genet. Dev. 31, 57-66 (2015).

3. Brinkman, A. B. et al. Histone modification patterns associated with the human X chromosome. EMBO Rep. 7, 628-634 (2006).

4. Heard, E. et al. Methylation of Histone H3 at Lys-9 Is an early mark on the $\mathrm{X}$ chromosome during X inactivation. Cell 107, 727-738 (2001).

5. Plath, K. et al. Role of histone $\mathrm{H} 3$ lysine 27 methylation in $\mathrm{X}$ inactivation. Science 300, 131-135 (2003).

6. Sharp, A. J. et al. DNA methylation profiles of human active and inactive $\mathrm{X}$ chromosomes. Genome Res 21, 1592-1600 (2011).

7. Yasukochi, Y. et al. X chromosome-wide analyses of genomic DNA methylation states and gene expression in male and female neutrophils. Proc. Natl Acad. Sci. USA 107, 3704-3709 (2010).

8. Carrel, L. \& Willard, H. F. X-inactivation profile reveals extensive variability in X-linked gene expression in females. Nature 434, 400-404 (2005).

9. Cotton, A. M. et al. Analysis of expressed SNPs identifies variable extents of expression from the human inactive X chromosome. Genome Biol. 14, R122 (2013).

10. Cotton, A. M. et al. Landscape of DNA methylation on the X chromosome reflects $\mathrm{CpG}$ density, functional chromatin state and X-chromosome inactivation. Hum. Mol. Genet. 24, 1528-1539 (2014).

11. Carrel, L. \& Willard, H. F. Heterogeneous gene expression from the inactive $X$ chromosome: an X-linked gene that escapes $\mathrm{X}$ inactivation in some human cell lines but is inactivated in others. Proc. Natl Acad. Sci. 96, 7364-7369 (1999).

12. Zhang, Y. et al. Genes that escape $\mathrm{X}$-inactivation in humans have high intraspecific variability in expression, are associated with mental impairment but are not slow evolving. Mol. Biol. Evol. 30, 2588-2601 (2013).

13. Yang, F., Babak, T., Shendure, J. \& Disteche, C. M. Global survey of escape from X inactivation by RNA-sequencing in mouse. Genome Res. 20, 614-622 (2010).

14. Peeters, S. B., Cotton, A. M. \& Brown, C. J. Variable escape from Xchromosome inactivation: identifying factors that tip the scales towards expression. Bioessays 36, 746-756 (2014).

15. Blewitt, M. E. et al. SmcHD1, containing a structural-maintenance-ofchromosomes hinge domain, has a critical role in $\mathrm{X}$ inactivation. Nat. Genet. 40, 663-669 (2008).

16. Nozawa, R. S. et al. Human inactive X chromosome is compacted through a PRC2-independent SMCHD1-HBiX1 pathway. Nat. Struct. Mol. Biol. 20, 566-573 (2013).

17. Massah, S. et al. Epigenetic characterization of the growth hormone gene identifies SmcHD1 as a regulator of autosomal gene clusters. PLOS ONE 9, e97535 (2014)

18. Luijk, R., Goeman, J. J., Slagboom, E. P., Heijmans, B. T. \& van Zwet, E. W. An alternative approach to multiple testing for methylation QTL mapping reduces the proportion of falsely identified CpGs. Bioinformatics 31, 340-345 (2015).

19. Orru, V. et al. Genetic variants regulating immune cell levels in health and disease. Cell 155, 242-256 (2013).

20. Roederer, M. et al. The genetic architecture of the human immune system: a bioresource for autoimmunity and disease pathogenesis. Cell 161, 387-403 (2015).

21. Wang Zhao, W., Hastie, T., Owe, A. B., J. confounder adjustment in multiple hypothesis testing. arXiv:1508.04178 (2015).

22. Chen, K. et al. Genome-wide binding and mechanistic analyses of Smchd1mediated epigenetic regulation. Proc. Natl Acad. Sci. USA 112, E3535-E3544 (2015). 
23. Daxinger, L. et al. An ENU mutagenesis screen identifies novel and known genes involved in epigenetic processes in the mouse. Genome Biol. 14, R96 (2013).

24. Gendrel, A.-V. et al. Smchd1-dependent and -independent pathways determine developmental dynamics of $\mathrm{CpG}$ island methylation on the inactive X chromosome. Dev. Cell 23, 265-279 (2012).

25. Gendrel, A.-V. et al. Epigenetic functions of Smchd1 repress gene clusters on the inactive X chromosome and on autosomes. Mol. Cell. Biol. 33, 3150-3165 (2013).

26. Lemmers, R. J. et al. Digenic inheritance of an SMCHD1 mutation and an FSHD-permissive D4Z4 allele causes facioscapulohumeral muscular dystrophy type 2. Nat. Genet. 44, 1370-1374 (2012).

27. Falckenhayn, C. et al. Comprehensive DNA methylation analysis of the Aedes aegypti genome. Sci. Rep. 6, 36444 (2016).

28. Breiling, A. \& Lyko, F. Epigenetic regulatory functions of DNA modifications: 5-methylcytosine and beyond. Epigenetics Chromatin 8, 24 (2015).

29. Gilbert, W. V., Bell, T. A. \& Schaening, C. Messenger RNA modifications: form, distribution, and function. Science 352, 1408 LP-1401412 (2016).

30. Wu, T. P. et al. DNA methylation on N6-adenine in mammalian embryonic stem cells. Nature 532, 329-333 (2016).

31. Kundaje, A. et al. Integrative analysis of 111 reference human epigenomes. Nature 518, 317-330 (2015).

32. Mould, A. W. et al. Smchd1 regulates a subset of autosomal genes subject to monoallelic expression in addition to being critical for $\mathrm{X}$ inactivation. Epigenetics Chromatin 6, 19 (2013).

33. Chu, C. et al. Systematic Discovery of Xist RNA Binding Proteins. Cell 161, 404-416 (2018).

34. Sato, T., Okumura, F., Ariga, T. \& Hatakeyama, S. TRIM6 interacts with Myc and maintains the pluripotency of mouse embryonic stem cells. J. Cell Sci. 125, 1544-1555 (2012).

35. Vaquerizas, J. M., Kummerfeld, S. K., Teichmann, S. A. \& Luscombe, N. M. A census of human transcription factors: function, expression and evolution. Nat. Rev. Genet. 10, 252-263 (2009).

36. Mason, A. G. et al. SMCHD1 regulates a limited set of gene clusters on autosomal chromosomes. Skelet. Muscle 7, 12 (2017).

37. Chess, A. Monoallelic expression of protocadherin genes. Nat. Genet. 37, 120-121 (2005).

38. van Greevenbroek, M. M. J. et al. The cross-sectional association between insulin resistance and circulating complement $\mathrm{C} 3$ is partly explained by plasma alanine aminotransferase, independent of central obesity and general inflammation (the CODAM study). Eur. J. Clin. Invest 41, 372-379 (2011).

39. Tigchelaar, E. F. et al. Cohort profile: LifeLines DEEP, a prospective, general population cohort study in the northern Netherlands: study design and baseline characteristics. BMJ Open 5, e006772 (2015).

40. Schoenmaker, M. et al. Evidence of genetic enrichment for exceptional survival using a family approach: the Leiden Longevity Study. Eur. J. Hum. Genet. (2005). https://doi.org/10.1038/sj.ejhg.5201508

41. Boomsma, D. I. et al. Netherlands Twin Register: A Focus on Longitudinal Research. Twin Res. 5, 401-406 (2002).

42. Hofman, A. et al. The Rotterdam Study: 2014 objectives and design update. Eur. J. Epidemiol. 28, 889-926 (2013).

43. Huisman, M. H. et al. Population based epidemiology of amyotrophic lateral sclerosis using capture-recapture methodology. J. Neurol. Neurosurg. Psychiatry 82, 1165-1170 (2011).

44. Deelen, J. et al. Genome-wide association meta-analysis of human longevity identifies a novel locus conferring survival beyond 90 years of age. Hum. Mol. Genet. 23, 4420-4432 (2014).

45. Lin, B. D. et al. The genetic overlap between hair and eye color. Twin Res. Hum. Genet. 19, 595-599 (2016)

46. Consortium, T. G. et al. Whole-genome sequence variation, population structure and demographic history of the Dutch population. Nat. Genet. 46, 818-825 (2014).

47. Deelen, P. et al. Genotype harmonizer: automatic strand alignment and format conversion for genotype data integration. BMC Res. Notes 7, 901 (2014).

48. HowieB. N., DonnellyP., \& MarchiniJ. A flexible and accurate genotype imputation method for the next generation of genome-wide association studies.Plos Genet. 5, e1000529 (2009).

49. Zhernakova, D. V. et al. Identification of context-dependent expression quantitative trait loci in whole blood. Nat. Genet. 49, 139-145 (2017).

50. Martin, M. Cutadapt removes adapter sequences from high-throughput sequencing reads. EMBnet. J. 17, 10 (2011).

51. Joshi Fass, J., N. Sickle: a sliding-window, adaptive, quality-based trimming tool for FastQ files (version 1.33). (2011).

52. Dobin, A. et al. STAR: ultrafast universal RNA-seq aligner. Bioinformatics 29, 15-21 (2013)
53. Tobi, E. W. et al. Early gestation as the critical time-window for changes in the prenatal environment to affect the adult human blood methylome. Int J. Epidemiol. 44, 1211-1223 (2015).

54. Chen, Y. A. et al. Discovery of cross-reactive probes and polymorphic CpGs in the Illumina Infinium HumanMethylation450 microarray. Epigenetics 8 203-209 (2013).

55. van Iterson, $M$. et al. MethylAid: visual and interactive quality control of large Illumina 450k datasets. Bioinformatics 30, 3435-3437 (2014).

56. Fortin, J. P. et al. Functional normalization of $450 \mathrm{k}$ methylation array data improves replication in large cancer studies. Genome Biol. 15, 503 (2014).

57. Aryee, M. J. et al. Minfi: a flexible and comprehensive Bioconductor package for the analysis of Infinium DNA methylation microarrays. Bioinformatics 30 1363-1369 (2014)

58. Gaunt, T. R. et al. Systematic identification of genetic influences on methylation across the human life course. Genome Biol. 17, 61 (2016)

59. Min, J., Hemani, G., Davey Smith, G., Relton, C. L. \& Suderman, M. Meffil efficient normalisation and analysis of very large DNA methylation samples. Doi.Org 125963 (2017). https://doi.org/10.1101/125963

60. Datta, S. R. et al. A threonine to isoleucine missense mutation in the pericentriolar material 1 gene is strongly associated with schizophrenia. Mol. Psychiatry 15, 615 (2008).

61. Hannon, E. et al. An integrated genetic-epigenetic analysis of schizophrenia evidence for co-localization of genetic associations and differential DNA methylation. Genome Biol. 17, 176 (2016).

62. Consortium, T. I. S. Rare chromosomal deletions and duplications increase risk of schizophrenia. Nature 455, 237-241 (2008).

63. Moayyeri, A., Hammond, C. J., Valdes, A. M. \& Spector, T. D. Cohort Profile: TwinsUK and Healthy Ageing Twin Study. Int. J. Epidemiol. 42, 76-85 (2013).

64. Andrew, T. et al. Are twins and singletons comparable? A study of diseaserelated and lifestyle characteristics in adult women. Twin Res. 4, 464-477 (2001).

65. Ikram, M. A. et al. The Rotterdam Study: 2018 update on objectives, design and main results. Eur. J. Epidemiol. 32, 807-850 (2017).

66. Simes, R. J. An improved Bonferroni procedure for multiple tests of significance. Biometrika 73, 751-754 (1986).

67. Liptak, T. On the combination of independent tests. Magy. Tud. Akad. Mat Kut. Int. Kozl. 3, 171-197 (1958).

68. Kent, W. J. et al. The Human Genome Browser at UCSC. Genome Res. 12, 996-1006 (2002).

69. Martens, J. H. A. \& Stunnenberg, H. G. BLUEPRINT: mapping human blood cell epigenomes. Haematologica 98, 1487-1489 (2013).

70. Consortium et al. An integrated encyclopedia of DNA elements in the human genome. Nature 489, 57-74 (2012).

\section{Acknowledgements}

This research was financially supported by several institutions: BBMRI-NL, a Research Infrastructure financed by the Dutch government (NWO, numbers 184.021 .007 and 184.033.111); the UK Medical Research Council; Wellcome (www.wellcome.ac.uk; [grant number $102215 / 2 / 13 / 2$ to ALSPAC]); the University of Bristol to ALSPAC; the UK Economic and Social Research Council (www.esrc.ac.uk; [ES/N000498/1] to CR); the UK Medical Research Council (www.mrc.ac.uk; grant numbers [MC_UU_12013/1, MC_UU_12013/2 to JLM, CR]); the Helmholtz Zentrum München - German Research Center for Environmental Health, which is funded by the German Federal Ministry of Education and Research (BMBF) and by the State of Bavaria; the Munich Center of Health Sciences (MC-Health), Ludwig-Maximilians-Universität, as part of LMUinnovativ; the Wellcome Trust, Medical Research Council, European Union (EU), and the National Institute for Health Research (NIHR)- funded BioResource, Clinical Research Facility, and Biomedical Research Centre based at Guy's and St Thomas' NHS Foundation Trust in partnership with King's College London. Samples were contributed by LifeLines, the Leiden Longevity Study, the Netherlands Twin Registry (NTR), the Rotterdam Study, the Genetic Research in Isolated Populations program, the Cohort on Diabetes and Atherosclerosis Maastricht (CODAM) study, the Prospective ALS study Netherlands (PAN), Avon Longitudinal Study of Parents and Children (ALSPAC), International Schizophrenia Consortium, Cooperative Health Research in the Ausburg Region (KORA), TwinsUK. We thank the participants of all aforementioned biobanks and acknowledge the contributions of the investigators to this study. This work was carried out on the Dutch national e-infrastructure with the support of SURF Cooperative.

\section{Author contributions}

Conceptualization, B.T.H., E.W.vZ., R.L., L.D., S.M.vdM.; Methodology, R.L., E.W.vZ.; Formal Analysis, R.L., H.W., C.K.W., E.H., E.C.M., J.L.M., P.M., M.M.N.; Resources, H.M., C.R., J.M., M.W., J.T.B., R.J., A.Z., L.F., P.tH., D.I.B., C.M.vD., M.vG., J.H.V., C.W., J.vM., P.E.S.; Writing - Original Draft, RL; Writing - Review \& Editing, R.L., B.T.H., 
E.W.vZ., L.D., R.J., A.Z., L.F., P.tH., D.I.B., C.M.vD., M.vG., J.H.V., C.W., J.vM., P.E.S.; Visualization, R.L., B.T.H.; Supervision, B.T.H., E.W.vZ.

\section{Additional information}

Supplementary Information accompanies this paper at https://doi.org/10.1038/s41467018-05714-3.

Competing interests: The authors declare no competing interests.

Reprints and permission information is available online at http://npg.nature.com/ reprintsandpermissions/

Publisher's note: Springer Nature remains neutral with regard to jurisdictional claims in published maps and institutional affiliations. (c) (i) Open Access This article is licensed under a Creative Commons Attribution 4.0 International License, which permits use, sharing, adaptation, distribution and reproduction in any medium or format, as long as you give appropriate credit to the original author(s) and the source, provide a link to the Creative Commons license, and indicate if changes were made. The images or other third party material in this article are included in the article's Creative Commons license, unless indicated otherwise in a credit line to the material. If material is not included in the article's Creative Commons license and your intended use is not permitted by statutory regulation or exceeds the permitted use, you will need to obtain permission directly from the copyright holder. To view a copy of this license, visit http://creativecommons.org/ licenses/by/4.0/.

(C) The Author(s) 2018

\section{BIOS Consortium}

Marian Beekman ${ }^{1}$, Ruud van der Breggen ${ }^{1}$, Joris Deelen ${ }^{1}$, Nico Lakenberg${ }^{1}$, Matthijs Moed ${ }^{1}$, H. Eka D. Suchiman ${ }^{1}$ Wibowo Arindrarto ${ }^{2}$, Peter van't Hof ${ }^{2}$, Marc Jan Bonder ${ }^{15}$, Patrick Deelen ${ }^{15}$, Ettje F. Tigchelaar ${ }^{15}$, Alexandra Zhernakova ${ }^{15}$, Dasha V. Zhernakova ${ }^{15}$, Jenny van Dongen ${ }^{16}$, Jouke J. Hottenga ${ }^{16}$, René Pool ${ }^{16}$, Aaron Isaacs ${ }^{17}$, Bert A. Hofman ${ }^{17}$, Mila Jhamai ${ }^{18}$, Carla J.H. van der Kallen ${ }^{19}$, Casper G. Schalkwijk ${ }^{19}$, Coen D.A. Stehouwer ${ }^{19}$, Leonard H. van den Berg ${ }^{20}$, Michiel van Galen ${ }^{2}$, Martijn Vermaat ${ }^{2}$, Jeroen van Rooij ${ }^{18}$, André G. Uitterlinden ${ }^{18}$, Michael Verbiest ${ }^{18}$, Marijn Verkerk ${ }^{18}$, P. Szymon M. Kielbasa ${ }^{21}$, Jan Bot $^{13}$, Irene Nooren ${ }^{23}$, Freerk van Dijk ${ }^{24}$, Morris A. Swertz ${ }^{24}$ \& Diana van Heemst ${ }^{25}$

\footnotetext{
${ }^{23}$ Present address: SURFsara, Amsterdam 1098 XG, The Netherlands. ${ }^{24}$ Genomics Coordination Center, University Medical Center Groningen, University of Groningen, Groningen 9713 AV, The Netherlands. ${ }^{25}$ Department of Gerontology and Geriatrics, Leiden University Medical Center, Leiden 2333 ZC, The Netherlands
} 